\title{
Schöfthaler, Traugott
}

Vom additiven Wertkonsens zur Bildung für das 21. Jahrhundert. Die internationale Entstehungsgeschichte des Globalen Lernens

ZEP : Zeitschrift für internationale Bildungsforschung und Entwicklungspädagogik 23 (2000) 3, S. 19-23

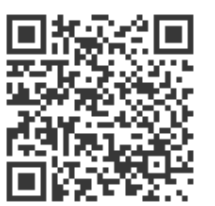

Quellenangabe/ Reference:

Schöfthaler, Traugott: Vom additiven Wertkonsens zur Bildung für das 21. Jahrhundert. Die internationale Entstehungsgeschichte des Globalen Lernens - In: ZEP : Zeitschrift für internationale Bildungsforschung und Entwicklungspädagogik 23 (2000) 3, S. 19-23 - URN:

urn:nbn:de:0111-pedocs-62535 - DOI: 10.25656/01:6253

https://nbn-resolving.org/urn:nbn:de:0111-pedocs-62535

https://doi.org/10.25656/01:6253

in Kooperation mit / in cooperation with:

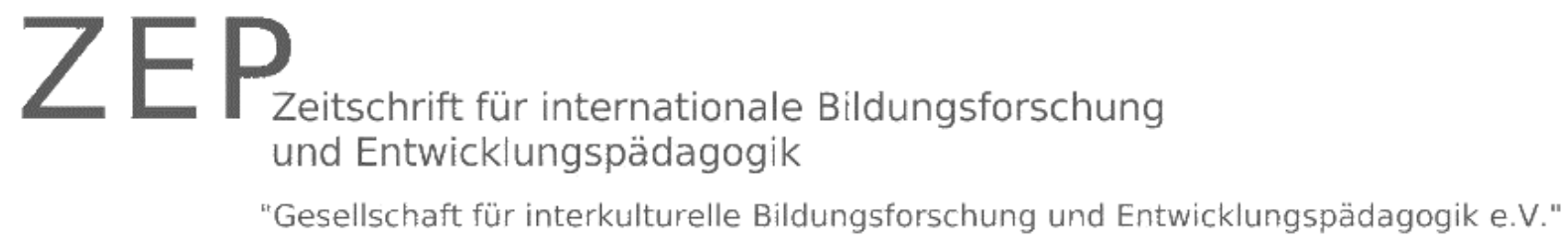

http://www.uni-bamberg.de/allgpaed/zep-zeitschrift-fuer-internationale-bildungsforschung-und-entwicklungspaedagogik/profil

\section{Nutzungsbedingungen}

Gewährt wird ein nicht exklusives, nicht übertragbares, persönliches und beschränktes Recht auf Nutzung dieses Dokuments. Dieses Dokument ist ausschließlich für den persönlichen, nicht-kommerziellen Gebrauch bestimmt. Die Nutzung stellt keine Übertragung des Eigentumsrechts an diesem Dokument dar und gilt vorbehaltlich der folgenden Einschränkungen: Auf sämtlichen Kopien dieses Dokuments müssen alle Urheberrechtshinweise und sonstigen Hinweise auf gesetzlichen Schutz beibehalten werden. Sie dürfen dieses Dokument nicht in irgendeiner Weise abändern, noch dürfen Sie dieses Dokument für öffentliche oder

kommerzielle Zwecke vervielfältigen, öffentlich ausstellen, aufführen, vertreiben oder anderweitig nutzen

Mit der Verwendung dieses Dokuments erkennen Sie die Nutzungsbedingungen an.

\section{Terms of use}

We grant a non-exclusive, non-transferable, individual and limited right to using this document.

This document is solely intended for your personal, non-commercial use. Use of this document does not include any transfer of property rights and it is conditional to the following limitations: All of the copies of this documents must retain all copyright information and other information regarding legal protection. You are not allowed to alter this document in any way, to copy it for public or commercial purposes, to exhibit the document in public, to perform, distribute or otherwise use the document in public.

By using this particular document, you accept the above-stated conditions of use.

\section{Kontakt / Contact:}

peDOCS

DIPF | Leibniz-Institut für Bildungsforschung und Bildungsinformation

Informationszentrum (IZ) Bildung

E-Mail: pedocs@dipf.de

Internet: www.pedocs.de

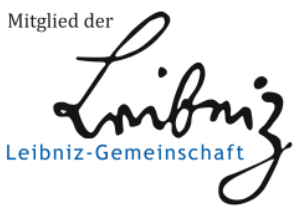




\section{Zeitschrift für internationale Bildungsforschung und Entwicklungspädagogik}

23. Jahrgang

David Selby

Klaus Seitz

Traugott

Schöfthaler

Klaus Karpen

Torsten Jäger

Jutta Janzen

Jos Schnurer

Uta Allers

Kommentar

BDW

VENRO

\author{
September
}

2000

ISSN 1434-4688D

\section{Global Education as Transformative Education}

Verlorenes Jahrzehnt oder pädagogischer Aufbruch? Zur Verankerung des Globalen Lernens 10 Jahre nach dem Kölner Bildungskongress Vom additiven Wertkonsens zur Bildung für das 21. Jahrhundert. Die internationale Entstehungsgeschichte des Globalen Lernens

24 Sind die deutschen Schulen fit für die Globalisierung?

26

Bildung 21. Lernen für eine gerechte und zukunftsfähige Entwicklung oder: Von der Notwendigkeit zu fragen, was wir hier eigentlich tun

29 Vom Süden lernen. Ein Interview mit Dorothea Giesche vom INKOTANetzwerk, Berlin

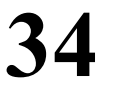

„Networking and Learning in a Changing World". Europaweite Aktionswoche zum Globalen Lernen für Schulen und Jugendgruppen

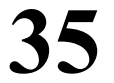
Wissenschaft für den Frieden. Bildung für Menschenrechte und Entwicklung. Das Internationale Jahr für eine Kultur des Friedens Mit Gemeinsamkeit und Differenz leben lernen. Über Erfahrungen mit dem NCBI-Ansatz in Berlin und Brandenburg

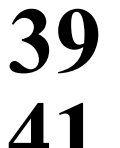

Nikolaus Schröck: Globales Lernen - Anspuch - Wirklichkeit - Vision

Nachwuchswissenschaftlerinnen in der Kommission BDW / Bericht über die 6. Arbeitstagung der AG Ethnopädagogik in der DGV

4.4 Bericht aus der AG Bildung im VENRO

45 Rezensionen / Kurzrezensionen / Unterrichtsmaterialien / Informationen

Impressum

ZEP - Zeitschrift für internationale Bildungsforschung und Entwicklungspädagogik 23. Jg. 2000, Heft 3

Herausgeber: Gesellschaft für interkulturelle Bildungsforschung und Entwicklungspädagogik e.V.

Schriftleitung: Annette Scheunpflug

Redaktionsanschrift: Katharina-Petersen-Weg 9, 30657 Hannover

Verlag: Verlag für Interkulturelle Kommunikation (IKO), Postfach 9004

21, 60444 Frankfurt/ Main, Tel.: 069/784808; ISSN 1434-4688 D

Erscheinungsweise und Bezugsbedingungen: erscheint vierteljährlich; Jahresabonnement DM 36,- Einzelheft DM 9,50; alle Preise verstehen sich zuzüglich Versandkosten; zu beziehen durch alle Buchhandlungen oder direkt vom Verlag. Abbestellungen spätestens acht Wochen vor $\mathrm{Ab}-$ lauf des Jahres.
Redaktion: Hans Bühler, Asit Datta, Georg-Friedrich Pfäfflin, Sigrid Görgens, Ulrich Klemm, Gregor Lang-Wojtasik, Claudia Lohrenscheit, Renate Nestvogel, Gottfried Orth, Bernd Overwien, Annette Scheunpflug, Klaus Seitz, Horst Siebert, Barbara Toepfer

Technische Redaktion: Gregor Lang-Wojtasik, 0511/814889. Abbildungen: (Falls nicht bezeichnet) Privatfotos oder Illustrationen der Autoren.

Titelbild: Indische Schulkinder (Foto: Gregor Lang-Wojtasik)

Diese Publikation ist gefördert vom Ausschußfür Entwicklungsbezogene Bildung und Publizistik, Stuttgart. Das Heft ist auf umweltfreundlichem chlorfreien Papier gedruckt. 
Zusammenfassung: Der Artikel beschreibt die Entstehungsgeschichte Globalen Lernens aus Perspektive der UNESCO. Globales Lernen ist ein Bildungskonzept geworden, das umfassend die Zukunftsfragen der Einen Welt reflektiert.

Mit der abschließenden Beratung eines mehrjährigen Projekts zur Erneuerung der staatsbürgerlichen Erziehung (,Education for Democratic Citizenship") durch die Bildungsministerkonferenz des Europarates im Oktober 2000 in Krakau gehen drei Jahrzehnte internationaler Bemühungen um Modernisierung der Bildung durch Aktualisierung von Zielen, Zwecken und Methoden von Bildung und Erziehung zu Ende. Begonnen hat dieser Prozeß mit Debatten der sechziger und frühen siebziger Jahre, die 1974 zu einem damals überraschenden Konsens der Regierungen aller UNESCO-Mitgliedstaaten führten. Der Konsens war eine Addition von Zielen, die in der Zeit des Kalten Krieges gleichzeitig die Funktion ideologischer Kampfbegriffe hatten: „Empfehlung über die Erziehung zu internationaler Verständigung und Zusammenarbeit und zum Frieden in der Welt sowie die Erziehung zur Achtung der Menschenrechte und Grundfreiheiten". Die Jahrzehnte dazwischen waren gesättigt mit Definitionsarbeit mehrerer Generationen von Ministerialbeamten und Pädagogen, Wissenschaftlern und Praktikern. Schon 1974 hat die UNESCO für den Alltagsgebrauch die Kurzform ,internationale Erziehung" gewählt, um ein Kernproblem zu bezeichnen: Nahezu alle Bildungssysteme haben ihre Wurzeln im Selbstverständnis von Nationalstaaten und deren Anspruch auf Definitionsmacht bei der Sozialisierung ihrer Subjekte. Dagegen hatten „Internationalisten" immer einen schweren Stand. Der in den letzten zehn Jahren zunehmend in den Vordergrund gerückte Begriff des „globalen Lernens" eignet sich für die dringend erforderliche Synthese schon wegen seiner Konnotation mit „Globalisierung". Selbst die am meisten auf Abgrenzung bedachten Regime appellieren an die internationale Gemeinschaft, wenn irgendwo im System der Vereinten Nationen über die Bewältigung der Auswirkungen von Globalisierung diskutiert wird. Den Windschatten dieser Konstellation haben zwei Weltkommissionen genutzt, um Pluralismus als Bildungsziel konsensfähig zu machen. Die fast überall in der Welt als Bedrohung empfundene Gefahr der Vereinheitlichung der Lebensweise erhebt Vielfalt zum grenzübergreifenden Wert. Diese Chance gilt es zu nutzen. Sie würde leichtfertig vertan, wenn der Begriff ,globales Lernen" zum Spielball neuerlichen Definitionsgerangels würde. Es geht um ein offenes Konzept, in das alle wichtigen neuen Ziele, Zwecke und Methoden von Bildung und Erziehung integriert werden können, die in den letzten drei Jahrzehnten formuliert und erprobt wurden. Als Konzept umfasst „globales Lernen" auch die wichtigsten Paradigmenwechsel der letzten Jahrzehnte: vom Lehren zum Lernen, von der biografisch abgrenzbaren Bildungs- und Ausbildungsphase zum lebensbegleitenden Lernen, vom Kanon der Lehrbücher zur Globalisierung der Wissensressourcen.

Ich schlage daher vor, das Bemühen um Begriffsklärung von globalem Lernen einzustellen. Es ist wichtiger, den in Jahrzehnten aufgehäuften Berg neuer Ziele, Zwecke und Methoden mit Orientierungsmarken zu versehen und Anregungen dort abzuholen, wo neue Erfahrungen gemacht wurden. Globales Lernen ist die Entwicklung und lebensbegleitende Nutzung der menschlichen Ressource Lernfähigkeit. Das „Globale" am Lernen ist sein Umfeld, das von keinem noch so weit entwickelten nationalen Bildungskanon mehr erfasst werden kann. Wir brauchen keine neuen Begriffsdefinitionen, sollten aber täglich den Perspektivenwechsel zwischen dem eigenen Erfahrungsraum und den Erfahrungsräumen anderer Menschen auf unserem Globus versuchen.

\section{Die Akkumulation neuer Bildungsziele und Methoden}

Die Verfassung der UNESCO aus dem Jahr 1945 formuliert „Erziehung zur Gerechtigkeit, zur Freiheit und zum Frieden" als globale Bildungsziele. Sie gelten als ,heilige Verpflichtung, die alle Völker im Geiste gegenseitiger Hilfsbereitschaft und Anteilnahme erfüllen müssen". Als eine der Ursachen des Zweiten Weltkriegs gilt die Verbreitung der Lehre eines unterschiedlichen Wertes von Menschen und Rassen ,unter Ausnutzung von Unwissenheit und Vorurteilen". Deshalb zählt die Verfassung eine Reihe weitere Aufträge an moderne Bildungssysteme auf: die Achtung der Menschenrechte und Grundfreiheiten, Völkerverständigung durch verbesserten Austausch von Informationen und Personen, Chancengleichheit und neue „Erziehungsmethoden, die am besten geeignet sind, die Jugend der ganzen Welt auf die Verantwortlichkeiten freier Menschen vorzubereiten".

Die beiden ersten Jahrzehnte des Kalten Krieges haben eine Präzisierung dieses umfassenden Modernisierungsprogramms verhindert. In dieser bleiernen Zeit schlugen auch alle Bemühungen fehl, die 1948 von der UNO-Generalversammlung verabschiedete Allgemeine Erklärung der Menschenrechte durch weitere Abkommen zu operationalisieren. Bei den Menschenrechten gelang der Durchbruch 1966 mit der Verabschiedung der grossen Pakte über wirtschaftliche und soziale, politische und kulturelle Rechte. Bei der Bil- 
dung dauerte es bis zum Jahr 1974, als die UNESCO-Generalkonferenz sich nach jahrelangen Debatten auf eine detaillierte „Empfehlung zur internationalen Erziehung" einigte. Im Tauwetter zwischen West und Ost war das jetzt möglich. Unter Hinzufügung des Grundsatzes der friedlichen Koexistenz zwischen unterschiedlichen sozialen und politischen Systemen wurden die Prinzipien aus dem Jahr 1945 bekräftigt. Die 45 Artikel umfassende Empfehlung enthält bereits fast alle Elemente, die heute unter dem Stichwort ,globales Lernen" in immer wieder neuen Varianten thematisiert werden. Zu den „Grundprinzipien" gehören die „Einführung der internationalen Dimension und globaler Sichtweisen auf allen Bildungsebenen und in allen Bildungsformen" ebenso wie das „Bewußtsein für die wachsende gegenseitige $\mathrm{Ab}$ hängigkeit zwischen Völkern und Nationen der Welt", aber auch methodische Prinzipien des Projektunterrichts wie „die Verknüpfung von Lernen, Ausbildung, Information und Aktion", die Vermittlung von sozialer Verantwortung und Solidarität, kritische Bewertung von Problemen im eigenen Land und im internationalen Kontext, Gruppenarbeit und freie Diskussion. Der ganze Text ist weitgehend eine Addition von Zielen, die damals im Westen und im Osten jeweils besonders hochgehalten wurden. Ein Beispiel: Unter der Überschrift „ethische und staatsbürgerliche Fragen" stehen als Empfehlungen Nr. 15 und 16 nebeneinander die beiden folgenden Sätze: „Ein Schwerpunkt der Erziehung soll die Frage nach den wirklichen Interessen der Völker sein, die mit den Interessen solcher Gruppen unvereinbar sind, die wirtschaftliche oder Machtmonopole innehaben und sie zur Ausbeutung und zur Kriegshetze einsetzen." Und: „Die Mitbestimmung von Schülern und Studenten bei der Organisation ihrer Lernbedingungen und ihrer Bildungseinrichtungen ist schon in sich selbst ein Element staatsbürgerlicher Bildung und somit ein wichtiger Faktor der internationalen Erziehung." Derart krasse Addition unverbundener Orientierungen lädt natürlich zum selektiven Lesen und damit zur faktischen Aufkündigung des Konsenses ein. Um so wichtiger war es, dass schon damals stellenweise die Synthese gelungen ist. So einigten sich die Regierungsdelegationen auf einen Katalog von sieben Weltproblemen, zu deren Lösung junge Menschen durch bessere - interdisziplinär angelegte Bildung befähigt werden sollten. Der Katalog umfasst, unterschiedlich detailliert, die folgenden Themen: Gleichberechtigung und Selbstbestimmungsrecht der Völker; Kriegsursachen und Voraussetzungen für die Erhaltung des Friedens; Menschenrechte, Rassismus und Kampf gegen Diskriminierung - die Rechte von Flüchtlingen werden besonders genannt; weltweite Wirtschaftsbeziehungen, Kolonialismus, soziale Entwicklung und Gerechtigkeit; Umweltverschmutzung und Erhaltung natürlicher Ressourcen; Bewahrung des kulturellen Erbes der Menschheit; und schließlich die Aufgaben des Systems der Vereinten Nationen bei der Bewältigung dieser Probleme.

Mehr als die Hälfte der 45 Empfehlungen enthalten das, was wir heute einen Katalog von „best practices" nennen würden, methodische und praktische Hinweise zur Umsetzung der Prinzipien und Verwirklichung der Ziele. Immer wieder findet sich hier die Forderung nach „Vermittlung globaler Sichtweisen" quer durch alle Stufen des Bildungs- systems, aber auch in der außerschulischen Bildung und in der Perspektive des lebenslangen Lernens. Für die Lehrerbildung werden fachübergreifende Ausbildungsgänge und Intensivkurse in der Vielfalt pädagogischer Methoden empfohlen. Lehrer sollen nicht nur ,ein fachübergreifendes Grundwissen über Weltprobleme und Fragen der internationalen Zusammenarbeit" erwerben, sondern ,gleichzeitig auch an Problemlösungen arbeiten". Die Lehr- und Lernmaterialien sollen das „Denken in globalen Zusammenhängen" fördern, Medienerziehung soll „den Schülern dabei helfen, die von den Massenmedien verbreiteten Informationen selektiv aufzunehmen und kritisch zu bewerten".

Zwanzig Jahre nach Verabschiedung der Empfehlung zur ,internationalen Erziehung" hatte die UNESCO-Generalkonferenz die Frage einer Revision der Empfehlung aus dem Jahr $1974 \mathrm{zu}$ entscheiden. Auf Antrag des vereinigten Deutschland und einer Reihe anderer Staaten aus Ost und West, Nord und Süd beschloss die Konferenz, den alten Text als Dokument eines unter schwierigen Bedingungen erreichten Konsens intakt und damit weiter in Geltung zu lassen. Das bedeutet zum Beispiel, dass alle Mitgliedstaaten die Pflicht haben, alle sechs Jahre über die Umsetzung zu berichten. Diese Berichte werden in Publikationen der UNESCO ausgewertet. Eine Synthese gibt beispielsweise der „UNESCO-Weltbildungsbericht 1995", der ein eigenes Kapitel zum Thema „Frieden, Achtung der Menschenrechte und demokratische Praxis als Bildungsziele" enthält.

Anstelle einer Neufassung der 1974er Empfehlung verabschiedete die UNESCO-Generalkonferenz einen „Integrierten Rahmenaktionsplan zur Erziehung für Frieden, Menschenrechte und Demokratie". Der Aktionsplan verbindet behutsam modernisierte Terminologie mit der Einführung der in den achtziger und frühen neunziger Jahren neu entstandenen Konsensformeln mit weltweitem Geltungsanspruch. So fügt Abschnitt 8 das Leitbild „Bürger einer pluralistischen Gesellschaft und einer multikulturellen Welt" in den Lernzielkatalog Frieden, Freundschaft und Solidarität zwischen Menschen und Völkern ein. Die Achtung des kulturellen Erbes und der Umweltschutz finden zusammen im Konzept einer langfristig tragfähigen, nachhaltigen Entwicklung. Weitere Synthesen versucht der Aktionsplan mit der Forderung neuer Programme für Lesen, Ausdrucksfähigkeit und Fremdsprachenunterricht, Prävention gegen das Schulversagen und für eine demokratische Schulverwaltung. Die Friedensthematik wird ergänzt um die Förderung der Fähigkeit zur gewaltlosen Konfliktlösung und um die historische Vertiefung des Sexismus-Problems. Das Ziel der kritischen Bewertung von Medieninformationen wird ergänzt um das der kritischen Mediennutzung - also umfassende Medienkompetenz. Weitere Rahmenvorgaben richten sich auf die Entwicklung von internationalen Netzwerken im Zusammenwirken von zwischenstaatlichen und Nichtregierungsorganisationen.

Die Selbtbescheidung der UNESCO auf Formulierung eines neuen Aktionsrahmens ist der Einstieg in das Verständnis des globalen Lernens als offenes Konzept. Der Rahmen grenzt andere normative Dokumente bewusst nicht aus, sondern lädt ein, über der Vielfalt einzelner Bildungsziele und pädagogischer Methoden den Blick aufs Ganze nicht zu ver- 
lieren. Deshalb hat die UNESCO-Generalkonferenz ohne Anspruch auf Vollständigkeit auch empfohlen, eine Reihe weiterer Empfehlungen, Deklarationen und Aktionspläne in die Bemühungen um eine umfassende Erneuerung des Bildungswesens zu integrieren - die Agenda 21 ebenso wie den 1993 verabschiedeten Weltaktionsplan für Menschenrechtserziehung, die Deklaration zum Internationalen Jahr für Toleranz 1995 ebenso wie andere Erklärungen und Aktionspläne der Vereinten Nationen zum Thema, zuletzt den Aktionsplan zum Internationalen Jahr 2000 für eine Kultur des Friedens und die anschließende Dekade für Frieden und Gewaltlosigkeit für die Kinder dieser Welt.

\section{Der Beitrag der Weltkommissionen „Bildung im 21. Jahrhundert" und „Kultur und Entwicklung"}

Einen Qualitätssprung auf dem Weg zu einem international konsensfähigen Konzept des globalen Lernens markieren die in den Jahren 1995 und 1996 beinahe zeitgleich veröffentlichten Berichte der Weltkommissionen „Bildung im 21. Jahrhundert" unter Leitung des ehemaligen Präsidenten der Europäischen Kommission Jacques Delors und „Kultur und Entwicklung" unter Leitung des ehemaligen UNOGeneralsekretärs Javier Pérez de Cuéllar. Unabhängig voneinander und gestützt auf ganz unterschiedliche wissenschaftliche und Erfahrungsressourcen kommen beide Berichte zu überraschend konvergenten Analysen und Schlußfolgerungen. Der Pérez-Report geht den Ursachen der zahlreichen bewaffneten Konflikte nach dem Ende des Kalten Krieges nach und stellt fest, welche Funktion dabei die internationalen Diskriminierungsverbote haben für Unterschiede der Sprache, kulturellen oder sozialen Herkunft, Religion oder anderer Überzeugung und politischer Meinung, Hautfarbe, Geschlecht, Rassenvorurteile. Anders als der Mainstream der aufklärerischen Tradition verfallt der Report jedoch an kaum einer Stelle in moralische Appelle an den Universalismus der Menschenrechte, sondern analysiert nach mehreren Seiten. So spricht er vom „Narzissmus der kleinen Unterschiede", mit dem Konfliktparteien beim Kampf um knappe Ressourcen immer wieder die Motivation ihrer Anhänger zur gewalttätigen Konfliktaustragung anfeuern. Gleichzeitig geht er hart ins Gericht mit jenen, die unter Berufung auf angeblich universelle abendländische Werte Interessenpolitik im globalen Maßstab kaschieren. Der Bericht mit dem programmatischen Titel „Unsere kreative Vielfalt" ist ein flammendes Plädoyer für die Erhaltung des überkommenen Pluralismus kultureller Orientierungen, aber gleichzeitig für die Wahlfreiheit des Einzelnen, der nicht zum Gefangenen kultureller Überlieferungen werden dürfe. An alte wie neue Nationalstaaten ergeht die Aufforderung, den Begriff der Nation von allen Konnotationen ethnischer, sprachlicher oder kultureller Exklusivität und Homogenität zu lösen. Folgerichtig geht der Pérez-Report über sein Mandat hinaus und widmet ein ganzes Kapitel der Situation von Kindern und Jugendlichen. Er plädiert für eine in jeder Hinsicht traditionskritische und in diesem Sinne traditionsbewußte Erziehung zum Leben in der multikulturellen Welt des 21. Jahrhunderts. Der Pérez-Bericht vollzieht den Paradigmenwechsel vom Lehren zum Lernen: Es sei falsch, das Bildungssystem als Transmissionsinstanz für staatlich fixierte Lehrplan- inhalte zu betreiben. „Kinder sind die Träger kultureller Traditionen, die sie mit früheren Generationen verbinden und die sie unablässig neu interpretieren und an ihre eigenen Bedürfnisse anpassen müssen, um damit die Grundlage für künftige kulturelle Innovation zu schaffen" (Pérez S. 164).

Der Delors-Bericht begnügt sich mit vergleichsweise knappen Analysen des Wandels von der lokalen Gemeinschaft zur Weltgesellschaft und vom sozialen Zusammenhang zur demokratischen Partizipation, um dann gleich zu den Bildungszielen zu kommen. Sein programmatischer Titel „Lernfähigkeit: Unser verborgener Reichtum" definiert Bildung als ein Ganzes, das von vier Lernsäulen getragen wird: Lernen, Wissen zu erwerben; Lernen zu handeln; Lernen für das Leben und - als Synthese - Zusammenleben lernen in einer multikulturellen Welt. Wie schon beim Pérez-Bericht steht im Mittelpunkt aller Aufforderungen an pädagogische Akteure die kulturelle Vielfalt, Delors spricht hier bewußt von einer „Erziehung zum Pluralismus", gemeint als Aufforderung an alle Akteure im Sozialisationsprozess, den Lernenden beim Einüben des Perspektivenwechsels zu helfen. Delors fordert die Ermunterung kindlicher Neugier, im naturwissenschaftlichen ebenso wie im Geschichts- und Sozialkundeunterricht: „Es sollte [...] die Rolle der Schule sein, jungen Menschen die historischen, kulturellen oder religiösen Hintergründe der verschiedenen Ideologien zu erklären, die um ihre Aufmerksamkeit kämpfen." (Delors S. 49) Der Delors-Bericht fordert Vermittlung von Pluralismus im Sinne einer aktiven Toleranz, die auf Interesse, Respekt und Wertschätzung anderer Menschen und Kulturen basiert.

\section{Internationale Erfahrungen und Projekte}

Die Entstehungsgeschichte des „globalen Lernens" als offenes Konzept hat sich natürlich nicht losgelöst von immer wieder neuen pädagogischen Erfahrungen ereignet. Die beiden genannten Berichte der Weltkommissionen und der UNESCO-Weltbildungsbericht 1995 greifen auf zahlreiche gute Praxisbeispiele zurück. Eine weitere Übersicht ist zu erwarten von der Veröffentlichung des Europaratsberichts zur Erneuerung der staatsbürgerlichen Bildung. Ich beschränke mich daher an dieser Stelle auf die kurze Vorstellung einiger typischer Projekte und Erfahrungen, die ich für besonders tragfähig halte.

Die UNESCO hat schon im Jahr 1953 das weltweite Netzwerk der UNESCO-Projekt-Schulen ins Leben gerufen. Es hat in schwierigen Zeiten Schulen aus der ganzen Welt unter einem Dach zusammengebracht und erfreut sich auch heute noch steigender Nachfrage. Mehr als 6000 Schulen aller Stufen in über 140 Ländern stellen sich der Herausforderung, über alle Grenzen hinweg globale Lerngemeinschaften aufzubauen. Die deutschen UNESCO-Projektschulen engagieren sich von Beginn an für das Ziel, internationalen Austausch anspruchsvoller zu gestalten als durch Planung von Klassenreisen in möglichst attraktive Länder. Dabei sind Ideen entstanden, die ebenso einfach und kreativ wie die folgende sind: Eine bayerische Berufsschule beschließt, die geplante Reise zur türkischen Partnerschule erst dann zu unternehmen, wenn ein Jahr Unterricht über den Islam und türkisch-osmanische Geschichte geschafft ist. Gleiches tut die Partnerschule mit der Aufnahme der Themen Christen- 
tum, deutsche und mitteleuropäische Geschichte in den Unterricht. Die Besonderheit des Vorhabens liegt in der Idee, die benutzten Unterrichtsmaterialien in die jeweils andere Sprache zu übersetzen und der Partnerschule mit der Bitte um Kommentar zu schicken. Die Kommentare werden zu neuen Unterrichtsthemen. Reziproker interkultureller Dia$\log$ an der Basis dürfte die Qualität persönlicher Begegnungen erheblich anheben.

Im Zeitalter zunehmender Verfügbarkeit des Internet ergeben sich hier ganz neue Möglichkeiten des kooperativen Lernens ohne Grenzen. Auf Anregung der Deutschen UNESCO-Kommission erproben derzeit mehr als hundert Schulen in Deutschland, Spanien, Kuba und anderen lateinamerikanischen Ländern die Erarbeitung von Unterrichtsthemen im Dialog zwischen jeweils zwei bis drei Schulen über ein ganzes Schuljahr hinweg. Das Ganze läuft in spanischer Sprache und bringt Leben in den Fremdsprachenunterricht an deutschen Schulen. Ich bin sicher, dass das bewußt für die deutschen Schulen eingebaute Handikap der Kommunikation in einer Fremdsprache weitere Wirkungen hat. Es ist inspiriert von Wolf Lepenies' programmatischer Aufforderung an die Deutschen, von einer Belehrungsgesellschaft $\mathrm{zu}$ einer Lerngesellschaft $\mathrm{zu}$ werden. Das gilt auch für das ambitionierte Projekt der internationalen Projekt tage der Solidarität, das getragen wird vom Netzwerk der UNESCO-Projektschulen, jedoch betont offen für die Beteiligung aller anderen Interessenten ist. Es wurde ins Leben gerufen von einer Gruppe von Lehrerinnen und Lehrern des Bielefelder Oberstufenkollegs zum 10. Jahrestag der Katastrophe von Tschernobyl am 26. April 1996. Aus der Verbindung von Solidaritätsaktion und Projektunterricht ist heute ein offenes weltweites Schulnetz geworden, das Aktivitäten über ein ganzes Schuljahr in allen Weltregionen verbindet. 1998 war der 50. Jahrestag der Allgemeinen Erklärung der Menschenrechte das Thema, in diesem Jahr geht es um „Bildung für nachhaltige Entwicklung". Mit der internationalen Koordination, heute zu einem erheblichen Teil über das Internet, hat die UNESCO erneut das Team am Oberstufenkolleg Bielefeld beauftragt. Als neues Element kommt eine UNESCO-Sommerschule hinzu, die das kooperative Lernen über Grenzen hinweg durch gemeinsame kreative Bildungsarbeit von Lehrern und Schülern ergänzt.

Ein anderes Projekt der UNESCO ist die Einrichtung eines „Bildungsservers" für die Länder des ehemaligen Jugoslawien in Sarajevo und Prishtina. Aus bitteren Erfahrungen selbst so mächtiger Institutionen wie der Weltbank und der EU mit dem Versuch, Schulbücher in der Region zu verbreiten, die dem Geist der Toleranz und Verständigung verpflichtet sind, entstand die Idee, in Zusammenarbeit mit örtlichen Nichtregierungsorganisationen Unterrichtsbausteine und Quellentexte in Serbisch, Kroatisch, Bosnisch und Albanisch über das Internet anzubieten. Seit März ist der Anfang mit Texten zum Thema Menschenrechte und Demokratie gemacht. Andere Themen werden folgen, so dass interessierte Lehrer und Lerner die Möglichkeit haben, das Schulbuchwissen aus dem Internet zu komplettieren. Solidaritätsaktionen wie die Einrichtung von Internet-Cafés im Kosovo, Computerspenden für bosnische Schulen und vor allem Lehrerfortbildungskurse stützen das Projekt ab.
Bei aller Begeisterung für die neuen Medien darf das Schulbuch nicht links liegen gelassen werden. Etwa gleichzeitig mit der Begründung des weltweiten Schulnetzwerks hat die UNESCO ihre Mitgliedstaaten immer wieder zu grenzüberschreitender Zusammenarbeit bei der Revision und Erneuerung der Schulbücher angeregt, insbesondere in den Fächern Geschichte, Geographie und Sozialkunde. Für Deutschland hat die Einrichtung gemeinsamer Schulbuchkommissionen mit anderen Ländern einen erheblichen Beitrag zur Aussöhnung mit den ehemaligen Kriegsgegnern und den Opfern des nationalsozialistischen Systems geleistet und tut dies auch heute noch. Besondere Wirkungen haben die seit 30 Jahren laufenden Arbeiten der deutsch-polnischen Schulbuchkommissionen gespielt, die auf deutscher Seite ebenso wie eine Reihe anderer bilateraler Kommissionen vom Georg-Eckert-Institut für internationale Schulbuchforschung in Braunschweig koordiniert werden. Dabei ist die Einsicht bei allen Beteiligten gewachsen, dass globales Lernen nicht in erster Linie durch Harmonisierung der Lehrbücher auf eine europäische oder weltweite Sichtweise gefördert wird. Es ist fast noch wichtiger, die historisch und kulturell gewachsenen unterschiedlichen Perspektiven wahrzunehmen und ihren Ursachen nachzugehen. Dass heutige Schulbücher so großen Wert auf Quellentexte unterschiedlicher Provenienz legen, ist ein wichtiges Ergebnis internationaler Schulbuchkooperation. Das Erschrecken über die Ursachen, den Verlauf und die Folgen der Kriege im ehemaligen Jugoslawien hat die UNESCO dazu motiviert, im September 1999 staatliche Lehrplangestalter, Schulbuchautoren und Pädagogen aus allen Staaten Südosteuropas zu einer Konferenz nach Visby einzuladen. In der Ruhe der schwedischen Insel Gotland haben sich wider Erwarten alle Teilnehmer auf gemeinsame Zielsetzungen für die „Entmilitarisierung" ihrer Schulbücher geeinigt. Im Mittelpunkt stehen die Förderung der Multiperspektivität durch Präsentation unterschiedlicher Quellentexte ohne vorgefertigte Interpretation, die Vermittlung der Handlungsperspektiven historischer und gegenwärtiger Akteure und die Forderung nach erweiterten Auswahlmöglichkeiten unter Lehr- und Lernmaterialien. Alle Länder, die internationale Schulbuchkommissionen eingerichtet haben, sind aufgefordert, ihre Erfahrungen mit den Ländern Südosteuropas zu teilen. So ehrenvoll derartige Bitten an deutsche Adressaten sind, sollte dieser Austausch doch vorrangig auf der neutralen Plattform internationaler Organisationen wie der UNESCO und des Europarates organisiert werden. Deutschland ist noch nicht angekommen auf dem Weg von einer Belehrungs- zu einer Lerngesellschaft. Die Deutsche UNESCO-Kommission verbindet deshalb die Beantwortung von Anfragen nach Beratung für israelischpalästinensische Schulbuchforschung oder bei der Lösung der Schulbuchfragen in Ostasien oder in Afrika mit dem Vorschlag, das gemeinsame Engagement unter dem Dach der UNESCO zu verstärken.

\section{Globalisierung gestalten}

Die Akkumulation neuer Bildungsziele in den letzten drei Jahrzehnten stand unter erheblichem Wertestress. Nahezu alle Bildungsziele von der UNESCO-Empfehlung zur internationalen Erziehung 1974 bis zum Europaratskonzept einer 
Erziehung demokratischer Staatsbürger im Jahr 2000 sind nicht nur aus der Kritik überholter Bildungskonzepte erwachsen, sondern aus der Erwartung, mit besserer Bildung auch etwas zur Verbesserung der Gesellschaft und der Welt insgesamt tun zu können. In der Praxis hatte dies erheblich überschießende Erwartungen an alle Akteure im Bildungsprozess zur Folge. Wer Frieden, Menschenrechte oder Nord-Süd-Beziehungen im Unterricht thematisierte, verstand sich selbst oder wurde von anderen verstanden als Akteur bei der Schaffung friedlicherer oder gerechterer Verhältnisse in der Welt. Daraus ist viel Frustration erwachsen, hat sich doch die Welt nicht in gleichem Maße friedlicher, toleranter und gerechter entwickelt, in dem diese Ziele im Unterricht thematisiert wurden.

Das Konzept globalen Lernens sollte daher nicht dem gleichen Wertestress ausgesetzt werden wie seine Vorgänger. So wie es guten und schlechten Unterricht nahezu unabhängig von der Thematisierung von Bildungszielen immer schon gegeben hat, wird es auch gutes und schlechtes globales Lernen geben. Das Konzept des globalen Lernens wäre nicht mehr hinreichend offen und synthesefähig, wenn es zu stark belastet würde mit dem Anspruch des Gutmenschentums. Ich halte es für ausreichend, die Ziele des globalen Lernens auf der Ebene zu bestimmen, auf der es um das Handlungspotential geht. Es erscheint einleuchtend, dass die Fähigkeit zum Perspektivenwechsel, zur Multiperspektivität, zur Akzeptanz von oder gar Neugier auf Meinungen, Werte oder Traditionen anderer Menschen, die sich mit den Zielen Pluralismus und Vielfalt verbindet, mehr Handlungspotential erschließt als reine Wissensakkumulation. Entlastung vom Wertestress könnte die betont sachliche Vermittlung des Themas Menschenrechte bringen. Es gibt rund 60 internationale Vereinbarungen über Menschenrechte und Grundfreiheiten. Ausweislich der Feststellungen der jeweils zuständigen, zumeist im Bereich der Vereinten Nationen angesiedelten Vertragskörperschaften gibt es keinen Staat, der für sich in Anspruch nehmen könnte, alle diese Vereinbarungen mustergültig umgesetzt zu haben. Auf Vorschlag der Deutschen UNESCO-Kommission wird die Bundesregierung demnächst alle ihre Berichte an diese internationalen Gremien und deren Kommentare hierzu veröffentlichen und diese Informationen laufend aktualisieren. Das schafft neue Möglichkeiten zum globalen Lernen und zur ,kritischen Bewertung von Problemen im eigenen Land und im internationalen Rahmen" (1974er Empfehlung Nr. 5). Es fördert die Einsicht, dass es auf der einen Seite internationale Vereinbarungen über Ziele einer gerechteren und besseren Welt im Detail gibt, und dass es auf der anderen Seite beharrlicher Bemühungen im eigenen Land wie weltweit bedarf, um diesen Zielen ein Stück näher zu kommen. Die Aktionskomponente ist fundamental für Globales Lernen. Es wäre jedoch fatal, Globales Lernen deshalb zum Politikersatz zu stilisieren. Im Fall des Gelingens dürfen wir jedoch erwarten, dass auch die Fähigkeit zunimmt, die Spannung zwischen Zielen und Zielverwirklichung nicht nur auszuhalten, sondern in Handlungsenergie umzusetzen. „Globalisierung gestalten" könnte ein Rahmen sein, in dem solche Lernziele formulierbar sind. Wenn die Verbesserung der Welt nicht mehr in erster Linie als Folge freier Entscheidungen von Menschen guten Wil- lens erwartet werden muss, sondern als Konsequenz aus der zunehmenden Dichte internationaler Vereinbarungen erwartet werden darf, mindert sich der Wertestress für Protagonisten des globalen Lernens erheblich. Im Jahr wurde 1945 die UNESCO gegründet als Staatenorganisation mit dem Auftrag, durch Förderung der internationalen Zusammenarbeit in Bildung, Wissenschaft, Kultur und Kommunikation zum Frieden und zur internationalen Sicherheit beizutragen. $\mathrm{Zu}$ diesem Auftrag gehört, das dabei wachsende Vertrauenskapital in internationale Vereinbarungen über Standards, gemeinsame Ziele und Verpflichtungen umzumünzen. Ich erhoffe mir von der Bildungsministerkonferenz des Europarats im Oktober 2000, dass sie beiden Dimensionen einer „Education for Democratic Citizenship" Auftrieb gibt: der weiteren Durchsetzung des Konzepts Globalen Lernens ebenso wie der Gestaltung der Globalisierung durch Staatsbürger, die ihre Regierungen zu mehr Engagement in den Vereinten Nationen drängen - nicht aus vermeintlich altruistischen Motiven, sondern aus vermehrter Einsicht in globale Zusammenhänge, die wir als Folge gelingenden globalen Lernens erwarten dürfen.

\section{Literatur:}

Allgemein im Internet: www.unesco.org; www.unesco.de; www.upsschulen.de.

Bloech, F./Lenzen, K. D./Nowotny, P./Strobl, G./Winter, F. (Hg.) Projekttag Tschernobyl. Internationale Schulkooperation zu einem Schlüsselproblem. Weinheim/Basel 1999.

Delors: Lernfähigkeit: Unser verborgener Reichtum. Bericht der Weltkommission Bildung für das 21. Jahrhundert (Präsident: Jacques Delors). Engl.-frz. Original Paris 1996. Dt.: Neuwied 1997.

Disarming History. International Conference on Combating Stereotypes and Prejudices in History-Textbooks of South-East Europe, Visby, Gotland (Sweden), 23.-25. September 1999. www.marebalticum.com/ disarminghistory

Europäisches Universitätszentrum für Friedensstudien, Deutsche UNESCO-Kommission, Österreichische UNESCO-Kommission (Hg.): Erziehung für Frieden, Menschenrechte und Demokratie im UNESCO-Kontext. Sammelband ausgewählter Dokumente und Materialien. Bonn 1997 (enthält u.a. die 1974er Empfehlung und den Rahmenaktionsplan von 1995 der UNESCO)

Hüfner, K./Reuther, W.(Hg.): UNESCO-Handbuch. Neuwied 1996 Perez: Our Creative Diversity. Report of the World Commission on Culture and Development. (President: Javier Pérez de Cuéllar). Paris/ Oxford $1995^{1}$, rev. $1996^{2}$

UNESCO-Weltbildungsbericht: Bonn 1996.

UNESCO/Georg-Eckert-Institute for international Textbook Research/ German Commission for UNESCO: Guidelines and Criteria for the Development, Evaluation and Revision of Curricula, Textbooks and other Educational Materials in International Education in Order to Promote an International Dimension in Education. Paris 1992 (dreisprachig englisch, französisch, spanisch).

Dr. Traugott Schöfthaler, geboren 1949, ist Generalsekretär der Deutschen UNESCO-Kommission, Bonn.

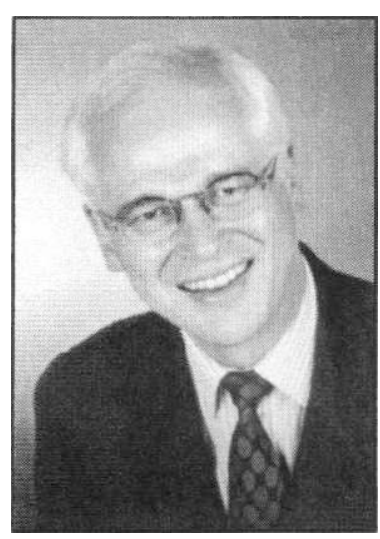

